02

\title{
Scaled quantum chemical studies and vibrational spectra of conjugated structure 2-benzylidenehydrazinecarbothioamide*
}

\author{
(C) P.V. Sreelaja and C. Ravikuma
}

Nanotechnology and Advanced Materials Research Centre,

Department of Physics, CMS College, Kottayam,

686001 Kerala, India

e-mail: rkr.ravi@gmail.com, ravikumar@cmscollege.ac.in

Received October 8, 2017; in final form, July 14, 2018

The title compound 2-Benzylidenehydrazinecarbothioamide was grown and the optimized molecular geometry was generated using density functional theory. Experimental Fourier Transform Raman and Infra red spectra were recorded and compared with the calculated results. The normal mode frequencies, Infra red and Raman intensities were computed with the Becke three Lee-Yang-Parr 6-31/ $\mathrm{G}^{*}$ method. Normal coordinate analysis is employed to analyze and interpret the vibrational assignments in the experimental spectra. The second harmonic efficiency of the grown crystal was studied by Kurtz and Perry powder reflection technique

DOI: $10.21883 /$ OS.2018.11.46814.223-17

* Полный текст статьи опубликован в английской версии журнала. 